\title{
The absence of a dense potential core in supercritical injection: A thermal break-up mechanism
}

Cite as: Phys. Fluids 28, 035103 (2016); https://doi.org/10.1063/1.4943038

Submitted: 31 August 2015 . Accepted: 18 February 2016 . Published Online: 08 March 2016

Daniel T. Banuti, and Klaus Hannemann

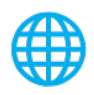

View Online

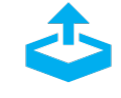

Export Citation

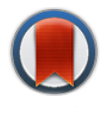

CrossMark

\section{ARTICLES YOU MAY BE INTERESTED IN}

Large-eddy simulation of nitrogen injection at trans- and supercritical conditions Physics of Fluids 28, 015102 (2016); https://doi.org/10.1063/1.4937948

Experiments and numerical simulation of mixing under supercritical conditions Physics of Fluids 24, 055104 (2012); https://doi.org/10.1063/1.3701374

Subcritical to supercritical mixing

Physics of Fluids 20, 052101 (2008); https://doi.org/10.1063/1.2912055

\section{AlP Advances Fluids and Plasmas Collection}




\title{
The absence of a dense potential core in supercritical injection: A thermal break-up mechanism
}

\author{
Daniel T. Banutia) and Klaus Hannemann \\ Spacecraft Department, German Aerospace Center (DLR), Institute of Aerodynamics \\ and Flow Technology, 37073 Göttingen, Germany
}

(Received 31 August 2015; accepted 18 February 2016; published online 8 March 2016)

\begin{abstract}
Certain experiments in quasi-isobaric supercritical injection remain unexplained by the current state of theory: Without developing a constant value potential core as expected from the mechanical view of break-up, density is observed to drop immediately upon entering the chamber. Furthermore, this phenomenon has never been captured in computational fluid dynamics (CFD) despite having become a de facto standard case for real fluid CFD validation. In this paper, we present strong evidence for a thermal jet disintegration mechanism (in addition to classical mechanical break-up) which resolves both the theoretical and the computational discrepancies. A new interpretation of supercritical jet disintegration is introduced, based on pseudo-boiling, a nonlinear supercritical transition from gas-like to liquid-like states. We show that thermal disintegration may dominate classical mechanical break-up when heat transfer takes place in the injector and when the fluid state is sufficiently close to the pseudo-boiling point. A procedure which allows to capture subsided cores with standard CFD is provided and demonstrated. ( 2016 AIP Publishing LLC. [http://dx.doi.org/10.1063/1.4943038]
\end{abstract}

\section{INTRODUCTION}

Supercritical injection is a topic of high technical relevance and ubiquity, being present in rocket engines, gas turbines, and Diesel engines. Initially, a supercritical pressure was obtained merely as a byproduct of seeking higher efficiency and power output by increasing operational pressure. Technology has now arrived at a point where the specifics of supercritical injection are explicitly sought to improve a combustion system (e.g., De Boer et al. ${ }^{1}$ ). In order to understand the expected gain, a comparison of sub- and supercritical injections is helpful.

In subcritical injection, the pressure of the combustion chamber is below the critical pressure of the injectant. Latent heat of vaporization and surface tension are finite. Figure 1(a) illustrates how instabilities in the shear layer form as waves with ever increasing amplitude until they disconnect from the main stream, featuring well defined sharp boundaries. ${ }^{2}$ The stripping of mass works its way towards the centerline of the central jet until the stream is no longer connected. The rate determining processes are atomization and vaporization. ${ }^{3}$

Contrast this to injection at supercritical pressures: Lacking surface tension and latent heat of vaporization, the subcritical break-up into ligaments and droplets is replaced by the turbulent mixing of the supercritical fluid with its surroundings, Fig. 1(b). Compared to low pressure injection, the interface looks diffuse, stringy features separate from the core, and the interface dissolves. ${ }^{3-5}$ As mixing and diffusion are faster processes than vaporization and surface tension inhibited break-up, supercritical mixing is expected to be faster and more effective. ${ }^{1}$

In any case, the break-up is caused by mechanical interaction in the shear layer. It commences upon entering the chamber and expands until it reaches the jet center line. This determines the length of the central stream core $L_{\mathrm{C}}$, also called dark core, liquid core, connected core, dense core, potential core, or unperturbed core. $L_{\mathrm{C}}$ is an important parameter: it is related to break-up efficacy,

a) Author to whom correspondence should be addressed. Electronic mail: dbanuti@ stanford.edu 


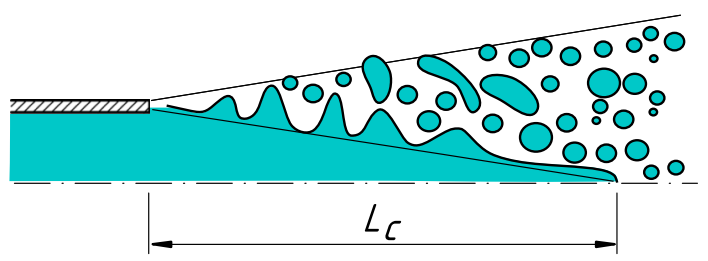

(a)

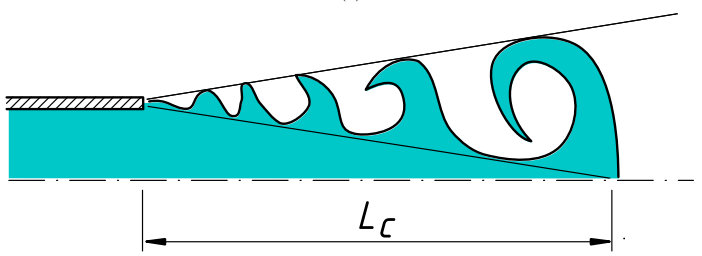

(b)

FIG. 1. Dense core length in sub- and supercritical injections. (a) Subcritical classical break-up. (b) Supercritical mixing-like disintegration.

it may be fundamental for combustion instabilities, ${ }^{6}$ and it is one of the few quantitative data actually measurable in high pressure injection systems. A number of correlations-experimental and theoretical—are suggested for $L_{\mathrm{C}}$ in the literature. Subscripts $\mathrm{G}$ for gaseous and $\mathrm{L}$ for liquid will be used for the surroundings and the central flow, respectively. This is done to interpret the correlations with respect to liquid injection applications. The liquid core length $L_{\mathrm{C}}$ is made dimensionless with the injector diameter $D$. For injection into stagnant environments

$$
\begin{aligned}
& L_{\mathrm{C}} / D=\text { const }, \\
& L_{\mathrm{C}} / D=f\left(\left(\rho_{\mathrm{L}} / \rho_{\mathrm{G}}\right)^{1 / 2}\right)
\end{aligned}
$$

are suggested. ${ }^{4,7-10}$

For cryogenic injection, these correlations have been determined in quasi-isobaric, laboratory scale injection experiments pioneered at the Air Force Research Laboratory (AFRL) by Chehroudi et al. ${ }^{11}$ and the German Aerospace Center (DLR) in Lampoldshausen by Oschwald and Schik, ${ }^{12}$ Oschwald and Micci. ${ }^{13}$ Furthermore, Branam and Mayer ${ }^{14}$ carried out a series of experiments which has become a canonical and mandatory test case for computational fluid dynamics (CFD) validation with real gas thermodynamics: Cryogenic nitrogen at sub- or supercritical temperature and supercritical pressure is injected into a chamber at supercritical pressure and ambient temperature. In addition to shadowgraphs and temperature, the density of the jet in the chamber has been measured quantitatively using Raman spectroscopy, yielding axial density profiles for a number of conditions.

These experiments have been simulated by numerous researchers (e.g., Kim et al., ${ }^{15}$ Müller et al. ${ }^{16}$ Niedermeier et al., ${ }^{17}$ Schmitt et al., ${ }^{18,19}$ Hickey et al. ${ }^{20}$ Cutrone et al.,${ }^{21,22}$ Cheng and Farmer, ${ }^{23}$ Jarczyk and Pfitzner, ${ }^{24}$ Terashima and Koshi, ${ }^{25}$ and Antunes et al. ${ }^{26}$ ). Exemplarily, two sets of results are shown in Fig. 2; the left and right plots show results for what Mayer et al. ${ }^{27}$ refer to as case $3\left(p=3.97 \mathrm{MPa}, T_{\text {in }}=126.9 \mathrm{~K}, u_{\text {in }}=4.9 \mathrm{~m} / \mathrm{s}\right)$ and case $4\left(p=3.98 \mathrm{MPa}, T_{\text {in }}=137.0 \mathrm{~K}\right.$, $u_{\text {in }}=5.4 \mathrm{~m} / \mathrm{s}$ ), respectively. The Reynolds average Navier Stokes (RANS) solution is from Mayer et al. ${ }^{27}$ the large eddy simulation (LES) stems from a high fidelity computation by Schmitt et al. ${ }^{19}$

Agreement between the simulations and the experiment is generally good for case 3, Fig. 2(a). For case 4, good agreement is found in the downstream part for $X / D>10$, cf. Fig. 2(b). However, a qualitative difference between CFD on one hand and the experiment on the other hand is visible immediately downstream of the injector: Instead of exhibiting a distinct dense core, as shown in CFD, the measured density is found to drop right upon entering the chamber. Other researchers who carried out CFD simulations of case 4 are Kim et al. ${ }^{15}$ Müller, ${ }^{16}$ Niedermeier, ${ }^{17}$ Terashima and Koshi, ${ }^{25}$ and Antunes et al. ${ }^{26} \mathrm{All}$ found a constant density core for case 4 . Thus, there exists a systematic discrepancy between CFD results and experimental data.

The axial density distribution of case 4 is no outlier, either. Figure 3 shows a compilation of all published axial density data of the campaign (Mayer et $a l .{ }^{27}$ ). Density data are only available 


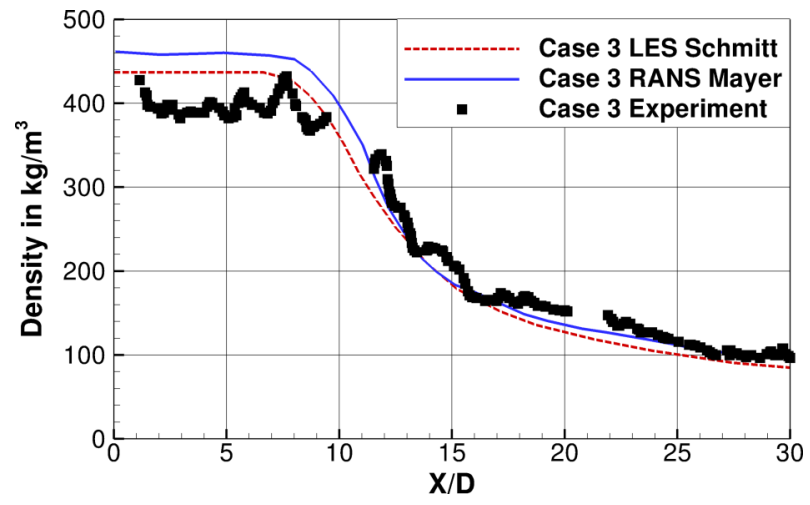

(a)

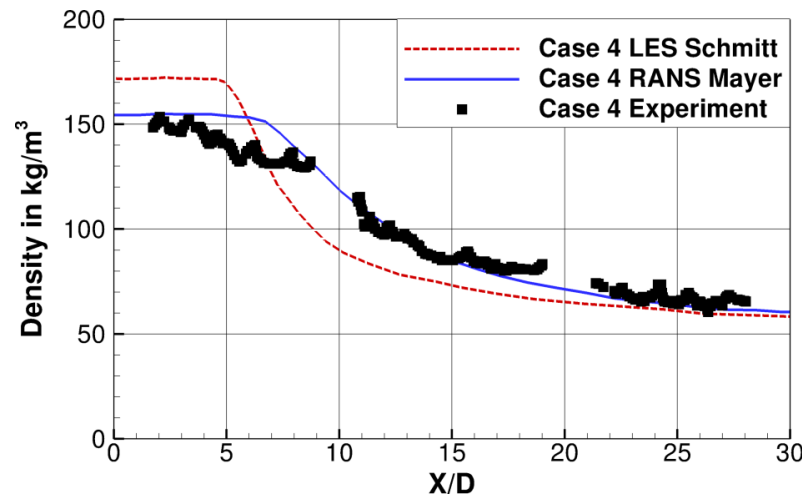

(b)

FIG. 2. Comparison of numerical and experimental axial density distributions in cryogenic nitrogen injection, case numbering according to Mayer $e t \mathrm{al}^{27}$ (a) $p=3.97 \mathrm{MPa}, T_{\text {in }}=126.9 \mathrm{~K}, u_{\text {in }}=4.9 \mathrm{~m} / \mathrm{s}$. (b) $p=3.98 \mathrm{MPa}, T_{\text {in }}=137.0 \mathrm{~K}$, $u_{\text {in }}=5.4 \mathrm{~m} / \mathrm{s}$.

for cases 3-10. The discussion in this paper is thus limited to these cases. Of the eight published distributions, only two exhibit a constant density core: cases 3 and 7. For all other cases, density can be seen to drop off immediately after injection.

A more thorough discussion of the experiment is in order. Experimental results have been published by Mayer, Tamura, Telaar, Branam, Hussong, and Schneider. ${ }^{14,27-30}$ A test matrix of nominal values $4 \mathrm{MPa}, 5 \mathrm{MPa}$, and $6 \mathrm{MPa}$ chamber pressure, $100 \mathrm{~K}, 120 \mathrm{~K}$, and $130 \mathrm{~K}$ injection temperature at $5 \mathrm{~m} / \mathrm{s}$ and $2 \mathrm{~m} / \mathrm{s}$ injection velocity has been investigated. The chamber was initially filled with nitrogen at room temperature.

As illustrated in Fig. 4, cryogenic nitrogen was fed through a $90 \mathrm{~mm}$ long injector of $2.2 \mathrm{~mm}$ diameter until the desired temperature was reached at the inflow to the injector (T1). At that point in time, the jet density was determined in the chamber using Raman spectroscopy through windows. In an additional experiment, a thermocouple was used to measure the jet temperature at the injector exit. Two temperatures were measured: T2a $1 \mathrm{~mm}$ outside of the exit plane with a thermocouple held perpendicular to the jet axis and T2b in the center of the jet, with a thermocouple held in parallel to the injector and positioned $1 \mathrm{~mm}$ inside the injector. The detailed test matrix is shown in Table I. Along with pressure $p$ and temperature $T$, a velocity $u$ is published which has been calculated from the mass-flow using the continuity equation. The density was calculated from temperature T1 and the chamber pressure using Younglove's modified Benedict-Webb-Rubin equation of state. $^{31}$

A discussion of temperature measurement error is in order, given the small differences between the measured values in Table I. While this is not offered by Branam and Mayer, ${ }^{28}$ the topic is treated thoroughly by Chehroudi et al. ${ }^{4}$ and Davis and Chehroudi ${ }^{32,33}$ for similar cryogenic nitrogen 


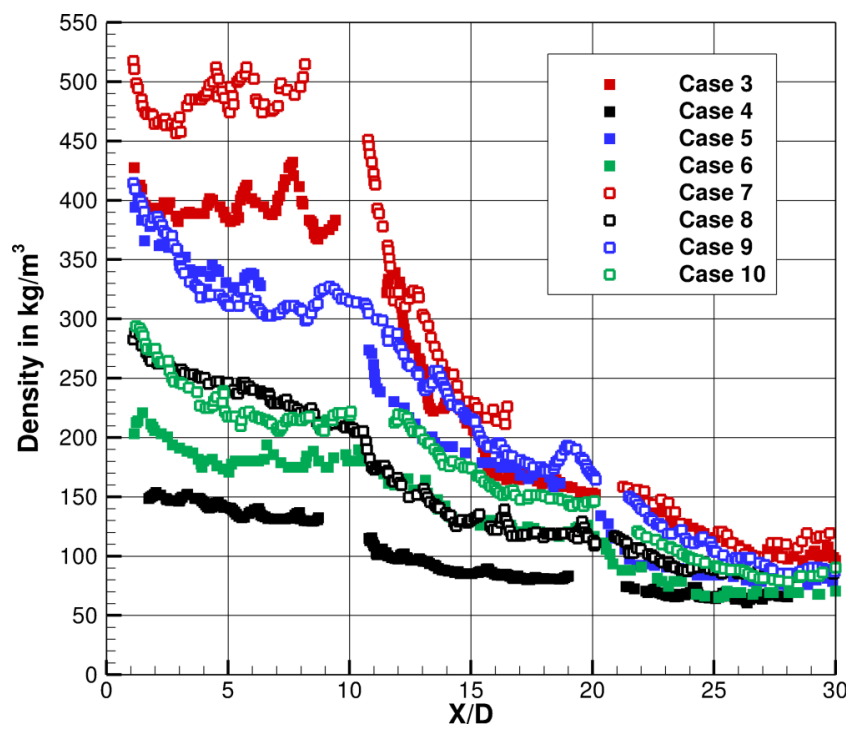

FIG. 3. Axial density distributions of nitrogen injection experiments by Mayer et ll. $^{27}$ and Branam and Mayer. ${ }^{28}$ case $^{2}$ numbering follows. ${ }^{27}$

injection experiments. Possible sources of error are the thermocouple touching the wall, ${ }^{32}$ the thermocouple distorting the flow field, ${ }^{32}$ a heterogeneous radial temperature profile, ${ }^{32}$ and production uncertainties (composition variation) in the thermocouple. ${ }^{33}$ They addressed the possible sources of error to improve the absolute accuracy. Using CFD analysis, they found that the influence of the flow field distortion by presence of the thermocouple is negligible. ${ }^{33} \mathrm{~A}$ temperature correction procedure allowed to estimate the centerline temperature from the measured bulk temperature by reducing the measured values by about $7 \mathrm{~K} .{ }^{32}$ Production uncertainties could be reduced to $\pm 0.8 \mathrm{~K}$ by recalibrating the thermocouples. ${ }^{33} \mathrm{All}$ in all, they found their results to be repeatable and concluded that while "the absolute magnitudes of the temperature measurements are in question here, the relative trends can be regarded as reliable." Then, even uncorrected measurements could be used to analyze the presented data. ${ }^{32}$ Thus we need to interpret the data in Table I likewise in the present paper, i.e., by comparing different cases.

Table II shows how the density in the injector drops for all cases as the nitrogen is slowly heated from T1 to T2a. Cases 3, 5, 7, and 9 enter the injector with similar densities exceeding $550 \mathrm{~kg} / \mathrm{m}^{3}$. The densities $\rho_{\mathrm{Tn}}$ have been obtained from the NIST database ${ }^{34}$ for the respective pressures and temperatures compiled in Table I.

This allows an evaluation of the expected liquid core length as given by Eq. (2). It is furthermore indicated whether a finite length dense core can be found in the experiment. With rising chamber pressures the chamber density grows, the square root of the density ratio decreases. The results do not clearly single out cases 3 and 7 as exhibiting the most pronounced dense cores: the density ratio with respect to $\rho_{\mathrm{T} 1}$ and $\rho_{\mathrm{T} 2 \mathrm{~b}}$ of case 5 actually exceeds that of case 7 . No indication for this can be seen in the axial density plots. Merely when T2a is picked as reference do the data predict a longer dense core for cases 3 and 7. However, no indication can be found as to why the dense core would collapse to naught in the other experiments.

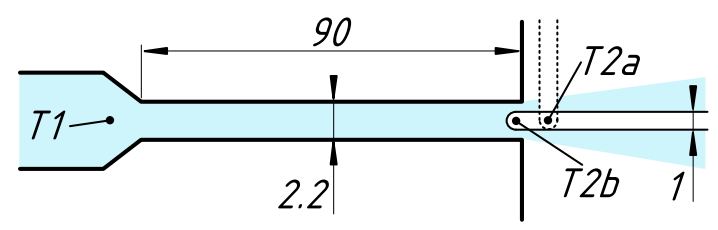

FIG. 4. Injector and temperature measurement details. Flow is from left to right. 
TABLE I. Test case specifications (Ref. 27, T1 from Ref. 28).

\begin{tabular}{lccccc}
\hline \hline Case & $\begin{array}{c}\text { Measured pressure } \\
\text { in MPa }\end{array}$ & $\begin{array}{c}\text { Computed velocity } \\
\text { in m/s }\end{array}$ & $\begin{array}{c}\text { Measured T1 } \\
\text { in K }\end{array}$ & $\begin{array}{c}\text { Measured T2a } \\
\text { in K }\end{array}$ & $\begin{array}{c}\text { Measured T2b } \\
\text { in K }\end{array}$ \\
\hline 3 & 3.97 & 4.9 & 120.9 & 126.9 & 122.9 \\
4 & 3.98 & 5.4 & 130.7 & 137.0 & 133.3 \\
5 & 4.90 & 2.0 & 120.7 & 131.0 & 122.6 \\
6 & 4.90 & 1.9 & 129.8 & 140.0 & 131.5 \\
7 & 5.01 & 4.5 & 120.7 & 126.2 & 122.5 \\
8 & 5.00 & 4.9 & 130.2 & 135.7 & 131.7 \\
9 & 5.85 & 2.0 & 120.7 & 135.0 & 122.8 \\
10 & 5.88 & 1.9 & 129.9 & 140.5 & 131.9 \\
\hline \hline
\end{tabular}

Thus, in addition to the CFD-experiment discrepancy, we find that correlations Eqs. (1) and (2), which have successfully been applied to a wide variety of injection cases, do not clearly single out the longest core cases. In fact, neither entrainment nor mass shedding, both of which rely on the image of a spreading mechanical interaction with the surrounding stream, can explain the complete absence of an unperturbed potential core, as seen in the experiments. Given the importance of the experiments as validation cases and the physical importance of the dense core length, this is somewhat disconcerting.

Several researchers have suggested that heat transfer may have an effect on jet disintegration. Indeed, heat transfer from the surrounding fluid has been identified to reduce supercritical spray penetration. ${ }^{35}$ Other researchers suggested that injector wall heat flux may play a role in jet disintegration. ${ }^{12,14,32,33}$ However, so far none have identified the subsided core as a fundamental violation of a mechanical break-up mode nor has this been investigated systematically.

In this paper, we will show that a purely mechanical view of supercritical injection is not sufficient, and discuss strong evidence for a disintegration mode which is thermal. Thermal disintegration resolves the discussed discrepancies and is unique for injection at supercritical pressures. We will show that it is related to pseudo-boiling, a supercritical state transition between liquid-like and gas-like states. We will furthermore demonstrate how standard real fluid CFD can capture this phenomenon.

\section{HEAT TRANSFER AND JET DISINTEGRATION}

\section{A. Heat transfer in the injector}

Mayer et $a l .{ }^{27}$ point out that heat transfer seems to have taken place in the injector, as the upstream temperature T1 is higher than either T2 measured farther downstream. Furthermore, Table I consistently shows that $\mathrm{T} 1>\mathrm{T} 2 \mathrm{~b}>\mathrm{T} 2 \mathrm{a}$ for all cases. As $\mathrm{T} 2 \mathrm{a}$ and $\mathrm{T} 2 \mathrm{~b}$ are measured at the injector

TABLE II. Densities and density ratios for measured temperatures. Data from NIST database. ${ }^{34}$

\begin{tabular}{lccccccc}
\hline \hline Case & $\begin{array}{c}\rho_{\mathrm{T} 1} \\
\text { in kg/m }\end{array}$ & $\begin{array}{c}\rho_{\mathrm{T} 2 \mathrm{a}} \\
\text { in kg/m }\end{array}$ & $\begin{array}{c}\rho_{\mathrm{T} 2 \mathrm{~b}} \\
\text { in kg/m }\end{array}$ & $\left(\rho_{T 1} / \rho_{\mathrm{c}}\right)^{\frac{1}{2}}$ & $\left(\rho_{T 2 a} / \rho_{\mathrm{c}}\right)^{\frac{1}{2}}$ & $\left(\rho_{T 2 b} / \rho_{\mathrm{c}}\right)^{\frac{1}{2}}$ & Dense core? \\
\hline 3 & 554.44 & 457.82 & 530.03 & 3.51 & 3.19 & 3.43 & Yes \\
4 & 252.40 & 164.37 & 195.83 & 2.36 & 1.91 & 2.08 & No \\
5 & 573.90 & 433.27 & 555.63 & 3.21 & 2.79 & 3.16 & No \\
6 & 458.31 & 224.71 & 421.46 & 2.87 & 2.01 & 2.75 & No \\
7 & 575.65 & 517.89 & 558.74 & 3.18 & 3.02 & 3.14 & Yes \\
8 & 456.64 & 315.07 & 425.38 & 2.84 & 2.36 & 2.74 & No \\
9 & 587.75 & 420.94 & 570.28 & 2.98 & 2.52 & 2.93 & No \\
10 & 498.13 & 320.80 & 471.73 & 2.73 & 2.19 & 2.66 & No \\
\hline \hline
\end{tabular}




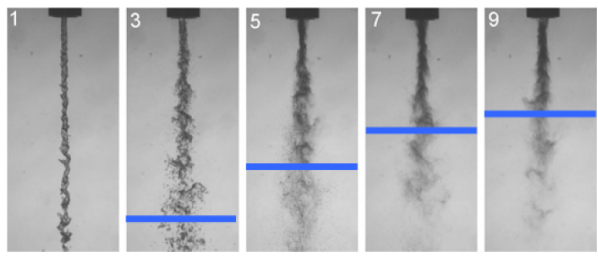

(a)

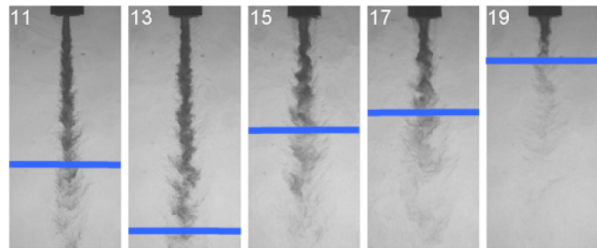

(b)

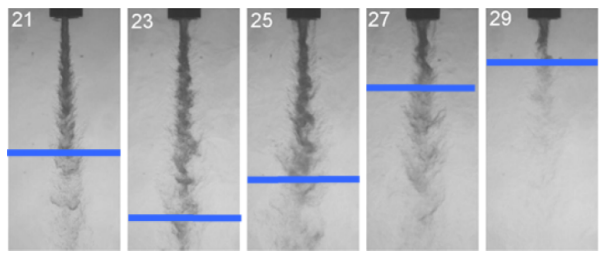

(c)

FIG. 5. Coaxial nitrogen injection shadowgraphs, modified from Chehroudi. ${ }^{36} p_{\mathrm{r}}$ is the chamber pressure reduced with the fluid critical pressure $p / p_{\text {cr }}$. Lines added to roughly indicate the position of maximum axial density gradient. Center mass flow constant, coaxial stream mass flow increases from left to right. Cases 1, 11, and 21 without gaseous co-flow. Reprinted with permission from B. Chehroudi, "Recent experimental efforts on high-pressure supercritical injection for liquid rockets and their implications," Int. J. Aerosp. Eng. 2012, 121802. Copyright 2012 Author(s), license under a Creative Commons Attribution License. (a) $p_{\mathrm{r}}=0.4$, (b) $p_{\mathrm{r}}=1.02$, and (c) $p_{\mathrm{r}}=1.4$.

exit and $\mathrm{T} 1$ at the beginning, the fluid seems indeed to have been heated while flowing through the injector. As depicted in Fig. 4, T2b has been measured in the center of the jet, inside the injector, parallel to the flow. It seems likely that the core temperature has been measured. T2a is rather unclear. It has been measured outside of the injector, with the probe held perpendicular to the flow. Thus, the probe may have entrained some of the ambient temperature fluid in the chamber, or the probe wires may have been heated. In both measured T2a and T2b, it seems likely that the flow has been perturbed significantly by the presence of the probes.

But can we conclude that this affects disintegration? While a number of experiments found evidence of heat flux affecting penetration, evidence for the existence and the significance of heat transfer in the injector is harder to come by. Davis and Chehroudi ${ }^{32,33}$ studied jet break-up of coaxial pure fluid jets, using liquid nitrogen in the center surrounded by a gaseous nitrogen stream. They varied the momentum flux ratio of the respective streams at different sub- and supercritical pressures and were particularly interested in the effect of acoustic excitation on injection. Figure 5 from Chehroudi $^{36}$ shows shadowgraphs of the break-up process for cases without acoustic excitation. The coaxial gaseous mass flow increases from left to right, the leftmost case does not have any. The added line roughly indicates a position of a high axial density gradient. Generally, a stronger co-flow enhances the break-up process, leading to shorter dense cores. However, the zero co-flow cases 11 and 21 at supercritical pressure in Figs. 5(b) and 5(c) show an earlier break-up than cases 13 and 23. The subcritical case 1 does not exhibit this behavior. This is counterintuitive and cannot be explained in terms of a purely mechanical view of jet break-up. Davis and Chehroudi investigated this thoroughly. They found that the coaxial annulus is filled with nitrogen at ambient temperature in the zero co-flow cases, leading to heat-up of the central liquid jet. This increased temperature was found to correlate with the shorter length dense core. So, while heat transfer was quantifiable, only qualitative shadowgraph data exist for the actual break up.

\section{B. Pseudo-boiling of supercritical fluids}

In Table I, we see that the temperature increase along the injector is very moderate: case 4 of Fig. 2(b) sees an increase from $\mathrm{T} 1=130.7 \mathrm{~K}$ to $\mathrm{T} 2 \mathrm{a}=137.0 \mathrm{~K}$ or $\mathrm{T} 2 \mathrm{~b}=133.3 \mathrm{~K}$, i.e., a $\Delta T$ of $6.3 \mathrm{~K}$ or $2.6 \mathrm{~K}$. Could this cause a qualitative change in disintegration characteristics at supercritical pressure, where no phase transition exists? The NIST database ${ }^{34,37}$ reveals that these minor 


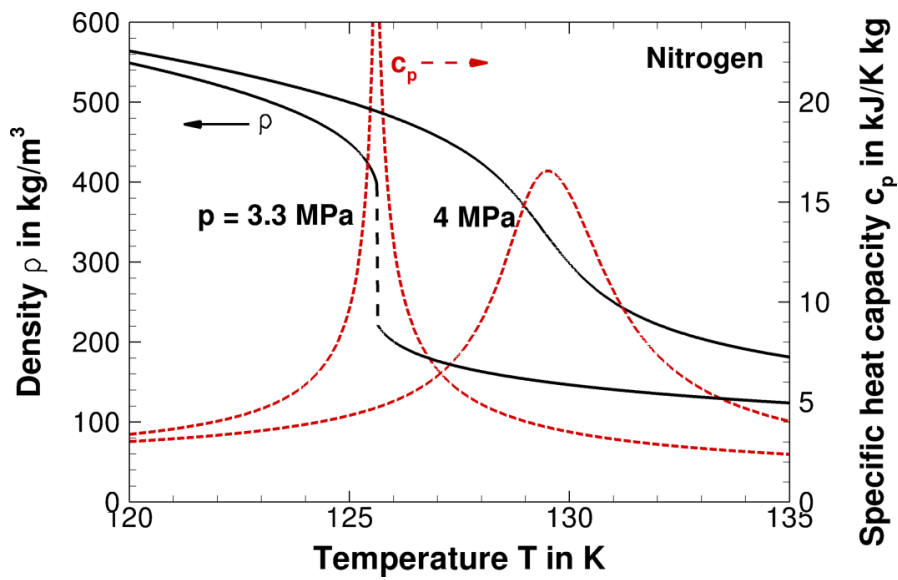

FIG. 6. Density and isobaric specific heat capacity for nitrogen at sub- (3.3 MPa) and supercritical (4 MPa) pressures.

changes in temperature correspond to a drop in density of $35 \%$ and $22 \%$, respectively! Heating of a supercritical fluid is apparently not as smooth as is generally assumed.

The unexpected large drop in density caused by a minor change in temperature suggests to look into supercritical fluid heating more closely. Thermal phenomena at supercritical pressures are intimately related to the thermodynamic state of the fluid. Oschwald and Schik ${ }^{12}$ pointed out that, at a given supercritical pressure, a peak in specific heat capacity can be found at a certain supercritical temperature. This peak coincides with a maximum of the thermal expansion coefficient. Figure 6 illustrates this for nitrogen at a sub- and a supercritical pressure. At a subcritical pressure (3.3 MPa), the density drops discontinuously at the boiling temperature. As the temperature remains constant while heat is added for the phase change, the heat capacity diverges. At supercritical pressure $(4 \mathrm{MPa})$, no discontinuities prevail. However, a distinct peak in specific heat capacity can be observed at the temperature where the density gradient is maximum.

Thus, an isobaric process passing through this temperature upon heat addition experiences only a moderate temperature rise during a substantial increase in specific volume. This has been dubbed "pseudo-boiling" by Oschwald et al..$^{5}$ for its resemblance with the subcritical phenomenon. The energy required to heat the fluid through the peak in specific heat was speculated to have influence on the dense core structure. Zong and Yang ${ }^{38}$ and Mayer et al. ${ }^{27}$ later reported similar findings. The term "pseudo-boiling" has first been used in the 1960s, initially introduced to describe supercritical phenomena which resemble subcritical surface boiling in heat transfer to pipes (Kafengauz and Federov ${ }^{39}$ ). However, as Oschwald and Schik ${ }^{12}$ pointed out, there is one fundamental difference: while the subcritical phase change occurs at constant saturation temperature, the supercritical transition sees an increase in enthalpy and temperature simultaneously.

A first quantitative investigation of the pseudo-boiling phenomenon has been carried out by Banuti. $^{40,41}$ It is found that the specific heat capacity distributions move to higher temperatures, widen, and flatten as the pressure is increased. In fact, a pseudo-boiling-line (PBL) can be constructed as an extension of the vapor-pressure curve at supercritical pressures. It follows the correlation:

$$
p_{\mathrm{r}}=\exp \left[\frac{T_{\mathrm{cr}}}{\theta_{\mathrm{cr}}}\left(T_{\mathrm{r}}-1\right)\right]
$$

where $T_{\mathrm{cr}}$ is the critical temperature and $\theta_{\mathrm{cr}}$ a fluid constant. The fraction $T_{\mathrm{cr}} / \theta_{\mathrm{cr}}$ is found to be approximately 5.5 for oxygen and nitrogen. ${ }^{40,41}$ Figure 7 shows a comparison between Eq. (3) and fluid data.

Banuti ${ }^{40,41}$ furthermore found that, at a reduced pressure of 3 , the $c_{p}$ peaks have flattened to the point that no nonlinear transition occurs anymore. The significance of the dramatic fluid property changes of pseudo-boiling are thus restricted to pressures $p_{\mathrm{cr}}<p<3 p_{\mathrm{cr}}$. 


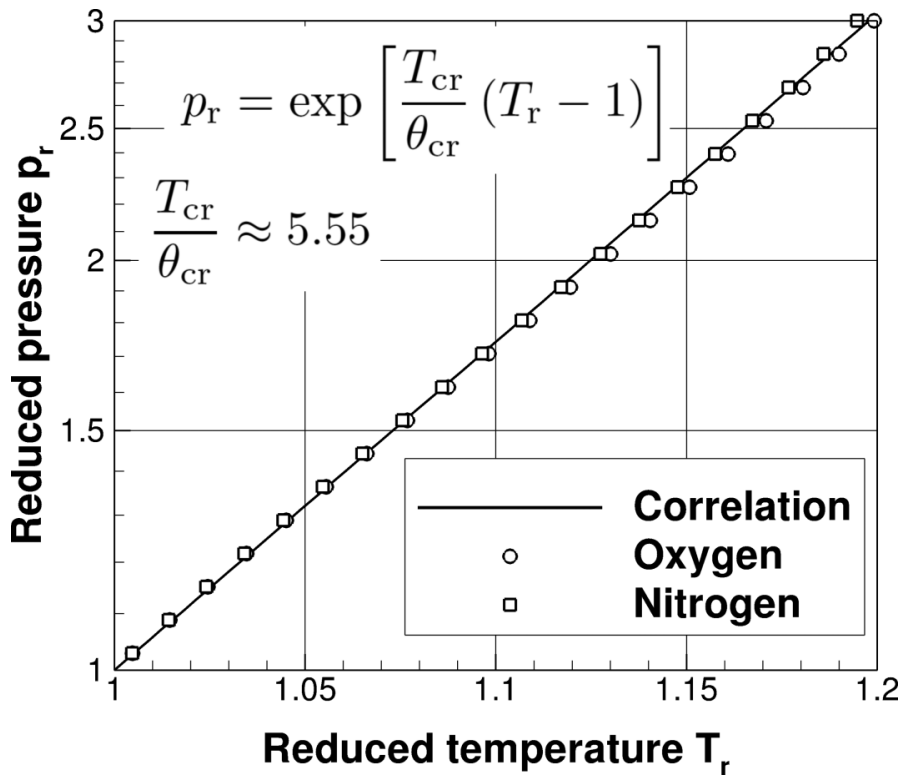

FIG. 7. Comparison of pseudo-boiling line correlation Eq. (3) (solid line) and maxima in specific heat capacity for oxygen and nitrogen from NIST ${ }^{34}$ (symbols).

Figure 8 shows the fluid state space in an extended $p$ - $T$ diagram. It compares the continuous but highly nonlinear variation between a liquid-like and a gas-like supercritical state to a subcritical vaporization process.

\section{Sensitivity to pseudo-boiling in injection}

We have now established a quantitative theoretical framework for pseudo-boiling. How can this be applied to the initial injection problem? We are looking for a state in which minor changes in temperature-e.g., caused by injector wall heat transfer-lead to large changes in density. The pseudo-boiling-line Eq. (3) fulfills this demand. Choosing the pseudo-boiling state furthermore has inherent thermodynamic meaning and is a unique condition. It is safe to assume that the more energy is required to heat a fluid to the pseudo-boiling state, the less sensitive it is to minor heat addition. Using pseudo-boiling theory, we can now exactly calculate this value: In order for a fluid to reach the pseudo-boiling state at constant pressure, the specific enthalpy of the fluid initially at $T_{\text {in }}$

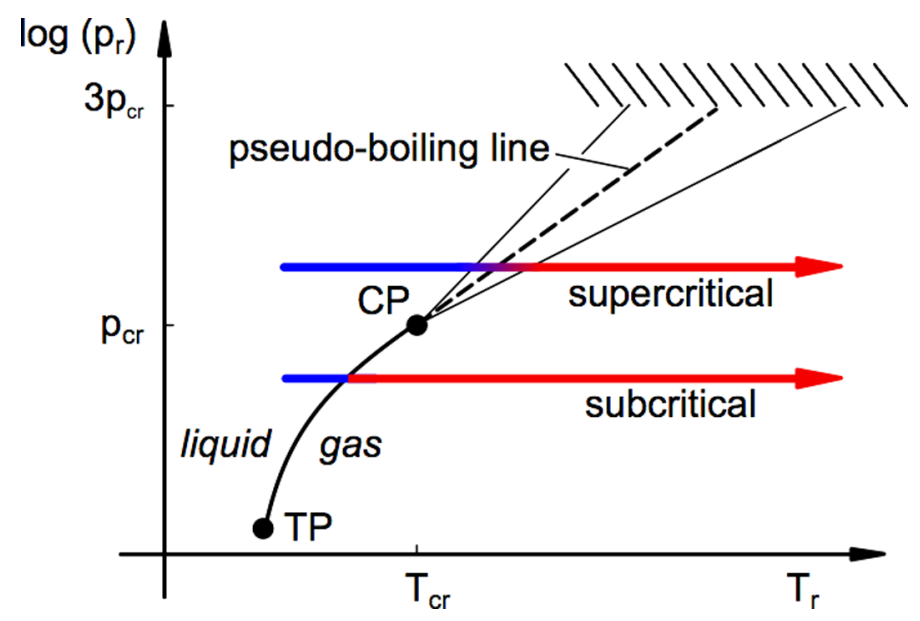

FIG. 8. Characteristics of supercritical injection. TP and CP denote triple point and critical point, respectively. Index "r" indicates reduced values which are nondimensionalized with the respective critical values. 


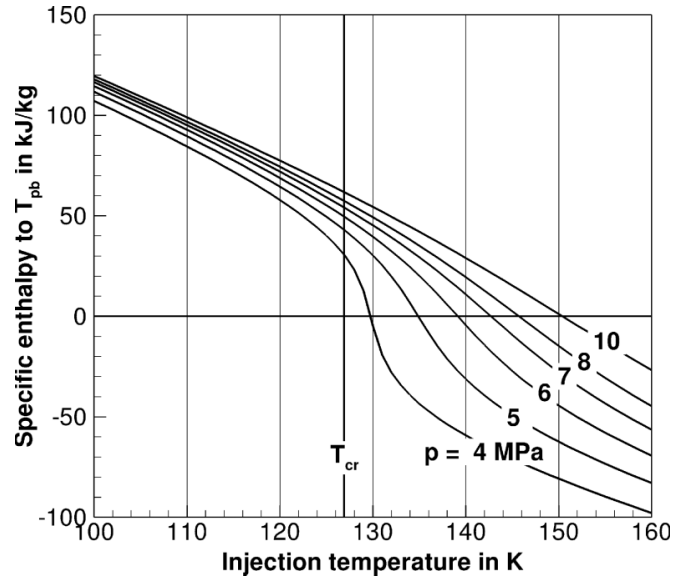

(a)

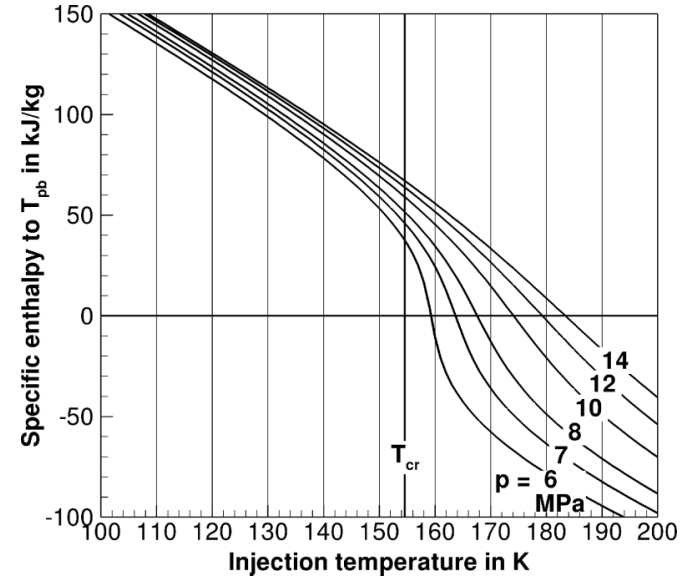

(b)

FIG. 9. Required pseudo-vaporization enthalpy for injection at different states. (a) Nitrogen. (b) Oxygen.

needs to be raised to reach $T_{\mathrm{pb}}$ by an amount

$$
\Delta h_{\mathrm{pb}}=\int_{T_{\mathrm{in}}}^{T_{\mathrm{pb}}} c_{p}(T) \mathrm{d} T,
$$

where index pb stands for pseudo-boiling. If it absorbs less heat than $\Delta h_{\mathrm{pb}}$, the fluid remains liquid-like; once it has absorbed more, it becomes gas-like. To transform a distinct mass $m$, e.g., of a droplet, thus requires the enthalpy

$$
\Delta H_{\mathrm{pb}}=m \int_{T_{\mathrm{in}}}^{T_{\mathrm{pb}}} c_{p}(T) \mathrm{d} T .
$$

The power required to do so continuously, e.g., in an injected stream with mass flow $\dot{m}$, is

$$
\Delta \dot{H}_{\mathrm{pb}}=\dot{m} \int_{T_{\mathrm{in}}}^{T_{\mathrm{pb}}} c_{p}(T) \mathrm{d} T .
$$

Equation (5) can be interpreted as an analogy to the latent heat of vaporization required to gasify, say, a supercritical droplet of mass $m$; Eq. (6) describes the power to gasify a liquid jet. With $m$ or $\dot{m}$ known, Eqs. (5) and (6) merely depend on the integral of Eq. (4). Figure 9 shows its evaluation for nitrogen and oxygen using NIST data. ${ }^{34}$ The initial temperature $T_{\text {in }}$ is shown on the abscissa, the ordinate is the required specific enthalpy $\Delta h_{\mathrm{pb}}$ to reach the pseudo-boiling state with the associated steep density gradient. Now, with injection temperature and chamber pressure known, $\Delta h_{\mathrm{pb}}$ can be determined.

Figure 9 has a number of interesting properties: The graphs for the different pressures intersect the ordinate zero line at the pseudo-boiling temperature of the respective pressure: Fig. 7 can thus be reconstructed from this diagram. It furthermore shows that fluid behavior close to the pseudo-boiling temperature at low supercritical pressures is very sensitive to temperature. Here, a minor change in temperature has a drastic influence on fluid enthalpy, and consequently, density. The degree of nonlinearity when passing through the pseudo-boiling temperature reduces at higher pressures. No significant dependence of heat capacity (i.e., slope of the graph) on temperature can be seen for pressures of $10 \mathrm{MPa}$ for nitrogen and $14 \mathrm{MPa}$ for oxygen, corresponding to reduced pressures of 2.9 and 2.8, respectively. This underlines the previous results of Banuti ${ }^{40,41}$ which state that pseudo-boiling effects are limited to reduced pressures lower than 3. Figure 9 is furthermore a striking demonstration that the critical temperature loses any significance as a transition marker at supercritical pressures: The steepest gradient is always centered around the pseudo-boiling temperature, no distinct changes occur at $T_{\text {cr. }}$. Finally, Fig. 9 reveals that for a given subcritical injection temperature, the required enthalpy difference grows when the pressure is increased. This is not 
obvious, as the $c_{p}$ peak is significantly reduced towards higher pressures. However, this is more than compensated by the increasing pseudo-boiling temperature to be reached with growing pressure.

\section{A thermal disintegration mechanism}

We can now return to the dense core length of Fig. 1. In injection with negligible pressure drop, the classical core length is a measure of how fast the expanding shear layer propagates towards the centerline. The potential core thus enclosed is a region which is not yet influenced by this mechanical interaction. Necessarily, it commences when the stream enters the chamber, as the interaction takes place between the injected stream and the surrounding fluid. The potential core concludes where mixing with the warmer background gas has reached the centerline, leading to a rapid deterioration of density. Density distributions which drop upon entering the chamber, cf. Fig. 3, hence cannot be explained or understood in this framework.

Pseudo-boiling theory now provides an alternative mechanism leading to a substantial drop in density, which does not require entrainment: heat transfer to the supercritical fluid. This mechanism will henceforth be called "thermal disintegration." A large density gradient can be expected when the pseudo-boiling temperature $T_{\mathrm{pb}}$ is approached. Pseudo-boiling reaching the centerline then may cause a measurable drop in density, as observed in the experiments.

The fundamental difference to mechanical break-up is that thermal disintegration may already take place in the injector, prior to entering the chamber.

If sufficient energy is added, the dense core is thus terminated because fluid gasification is complete before shear layers reach the centerline. If little energy is added to the injected fluid, the mechanical break-up is dominant. Ultimately, a parameter comparing energy required to reach pseudo-boiling, i.e., $\Delta \dot{H}_{\mathrm{pb}}$ in Eq. (6), to supplied energy $\dot{Q}$ assesses the likelihood of thermal disintegration during injection,

$$
\Lambda=\frac{\Delta \dot{H}_{\mathrm{pb}}}{\dot{Q}}, \quad \Lambda \rightarrow\left\{\begin{array}{l}
\ll 1: \text { thermal break-up } \\
\gg 1: \text { mechanical break-up }
\end{array} .\right.
$$

Unfortunately, no data of heat transfer $\dot{Q}$ exist and more experiments are clearly needed. Nevertheless, for a given experiment, comparing $\Delta \dot{H}_{\mathrm{pb}}$ for different conditions should provide a meaningful classification.

\section{E. The boundary of a supercritical fluid}

The discussion in Subsections II A-II D additionally suggests a way to define the boundary of a supercritical fluid. This concept requires some elaboration, as, without phase change, no supercritical liquid and gaseous states may coexist in equilibrium, no clear interface with surface tension forms. Nevertheless, Fig. 5 shows that, especially near the injector, both subcritical and supercritical jets are clearly distinguishable from their surroundings. As it mixes with the warm background gas, dense supercritical fluid dissolves. Limiting our discussion to pure fluid behavior, as in the investigations of Davis and Chehroudi ${ }^{33}$ or Branam and Mayer, ${ }^{28}$ we now know that the point at which the fluid density changes most dramatically is the pseudo-boiling state. Again in analogy to the subcritical fluid, one may thus understand the boundary of a supercritical jet or droplet as the position at which the density gradient is maximum. Pseudo-boiling may thus not only provide a new break-up mechanism - it also defines the boundary of a supercritical jet in a thermodynamically unambiguous and meaningful way.

\section{REVIEW OF THE EXPERIMENT}

Having established the concept and the criteria of a thermal disintegration mechanism, we may now return to the analysis of Branam's and Mayer's ${ }^{28}$ experiment. 


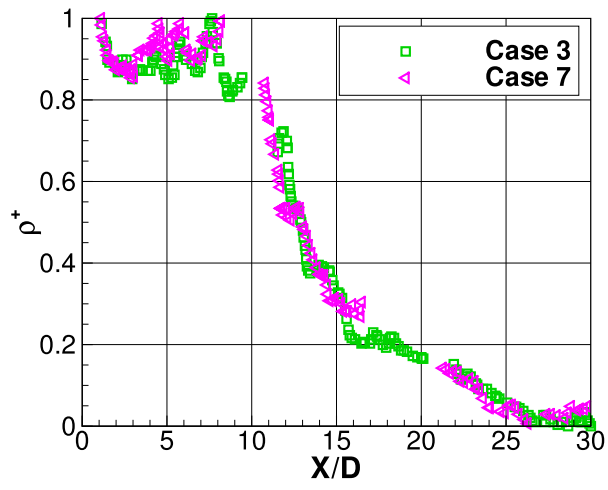

(a)

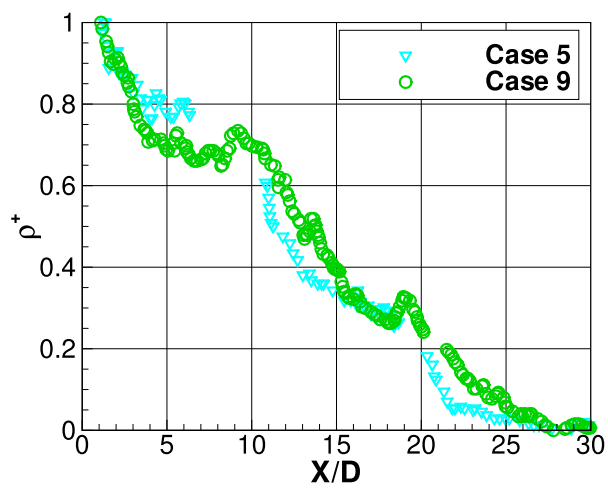

(c)

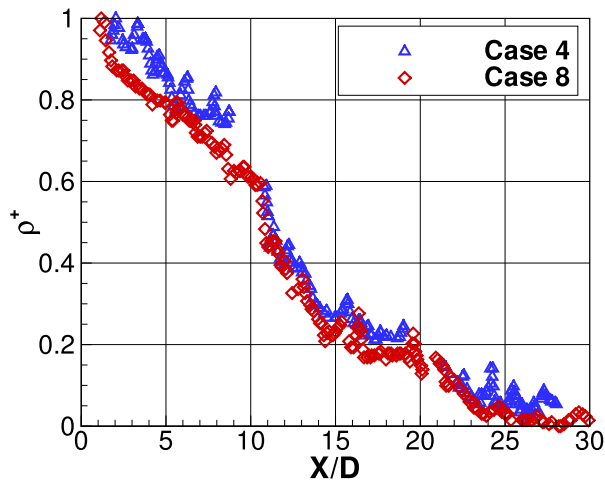

(b)

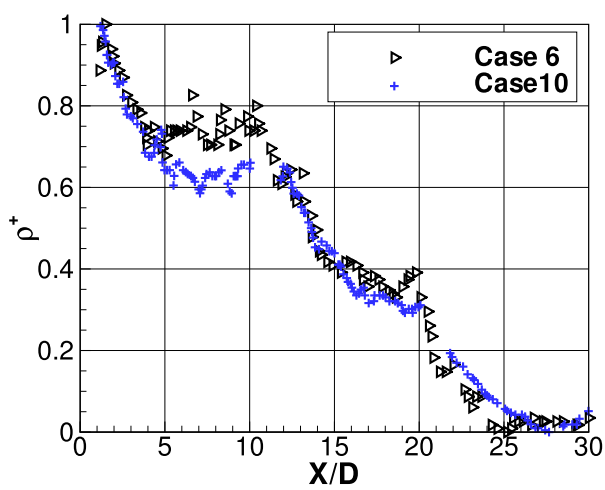

(d)

FIG. 10. Nondimensionalized axial density distributions, from Banuti and Hannemann. ${ }^{42}$ (a) $u=5 \mathrm{~m} / \mathrm{s}, T=120 \mathrm{~K}$. (b) $u=5 \mathrm{~m} / \mathrm{s}, T=130 \mathrm{~K}$. (c) $u=2 \mathrm{~m} / \mathrm{s}, T=120 \mathrm{~K}$. (d) $u=2 \mathrm{~m} / \mathrm{s}, T=130 \mathrm{~K}$.

\section{A. Analysis of the density distributions}

First of all, a more detailed analysis of the available density distributions is required. Figure 3 showed all published data at once, in dimensional form. To allow a deeper analysis, we nondimensionalize each measurement with the lowest and highest respective density, $\rho_{\text {low }}$ and $\rho_{\text {high }}$, using

$$
\rho^{+}=\frac{\rho-\rho_{\text {low }}}{\rho_{\text {high }}-\rho_{\text {low }}} .
$$

Grouping the plots in terms of injection velocity and temperature yields Fig. 10. The result is striking: a dense core, as expected from mechanical break-up, can only be seen in cases 3 and 7 , Fig. 10(a). All other cases show a drop in density right from injection.

Beside the classical dense core, as depicted in Fig. 11(a), two additional characteristic shapes can be distinguished: cases 4 and 8 show a sloped density distribution which drops off rapidly, see Fig. 11(b); cases 5, 6, 9, and 10 exhibit an intermediate plateau, as in Fig. 11(c).

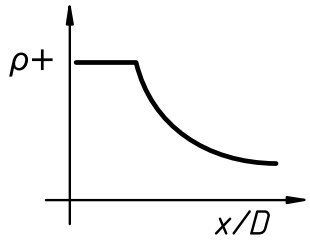

(a)

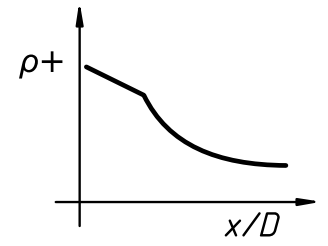

(b)

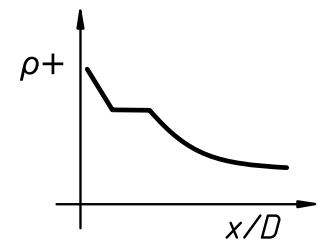

(c)

FIG. 11. Axial density distribution types. (a) Dense core. (b) Slope. (c) Plateau. 


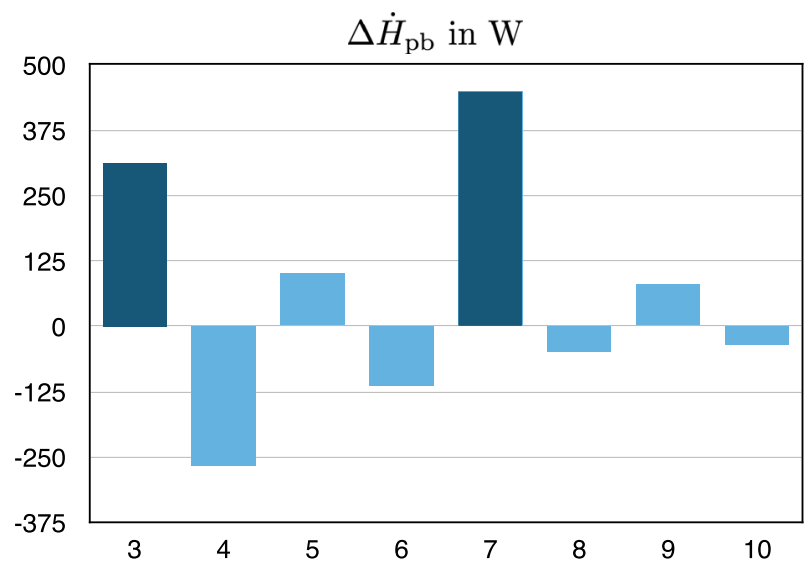

FIG. 12. Required pseudo-vaporization powers based on T2a.

Of the eight shown density distributions only two exhibit a dense core as expected from classical mechanical jet break-up theories. Thus, in the discussed campaign, the subsided core is not the outlier-the potential core is.

\section{B. Thermodynamic analysis}

Consider this from the perspective of pseudo-boiling. Figure 10 suggests that only two out of eight cases undergo a classical, mechanical break-up, all other cases show a dropping density upon entering the chamber. If this can be explained with a thermal mechanism, the existence of a constant density core should correlate with a high required thermal disintegration power $\Delta \dot{H}_{\mathrm{pb}}$ as defined in Eq. (6). First, the pseudo-boiling temperature $T_{\mathrm{pb}}$ for each pressure is given. Then, $\Delta \dot{H}_{\mathrm{pb}}$ is determined from each of the starting temperatures T1, T2a, T2b. We calculated the mass flow $\dot{m}$ from the density at $\mathrm{T} 1$ and chamber pressure, the injection velocity, and the injector cross section.

The result of this evaluation is presented in Fig. 12 exemplarily for a starting temperature T2a, a complete set of results is compiled in Table III. Disintegration types dense core, plateau, and sloped are denoted (c), (s), and (p), respectively.

We see that the pseudo-boiling condition successfully singles out the dense core cases 3 and 7 regardless of the chosen injection temperature $\mathrm{T} 1, \mathrm{~T} 2 \mathrm{a}$, or $\mathrm{T} 2 \mathrm{~b}$.

\section{CFD OF THERMAL DISINTEGRATION}

\section{A. Rationale}

The hypothesis of a thermal jet disintegration mechanism and its experimental and theoretical evidence has been discussed. Induced by heat flux inside the injector, this would lead to cryogenic

TABLE III. Thermal analysis of cryogenic injection experiments.

\begin{tabular}{lcrrrrr}
\hline \hline Case & $\begin{array}{c}T_{\mathrm{pb}} \\
\text { in K }\end{array}$ & $\begin{array}{c}\Delta \dot{H}_{\mathrm{bu}, \mathrm{T} 1} \\
\text { in W }\end{array}$ & $\begin{array}{c}\Delta \dot{H}_{\mathrm{bu}, \mathrm{T} 2 \mathrm{a}} \\
\text { in W }\end{array}$ & $\begin{array}{c}\Delta \dot{H}_{\mathrm{bu}, \mathrm{T} 2 \mathrm{~b}} \\
\text { in W }\end{array}$ & $\begin{array}{c}\dot{m} \\
\text { in g/s }\end{array}$ & Type \\
\hline 3 & 129.57 & 566.18 & 310.88 & 496.09 & 10.33 & $\mathrm{c}$ \\
4 & 129.63 & -90.39 & -265.30 & -190.34 & 5.18 & $\mathrm{~s}$ \\
5 & 134.41 & 270.63 & 103.59 & 246.35 & 4.36 & $\mathrm{p}$ \\
6 & 134.41 & 99.08 & -113.30 & 69.08 & 3.31 & $\mathrm{p}$ \\
7 & 134.93 & 616.73 & 448.72 & 565.50 & 9.85 & $\mathrm{c}$ \\
8 & 134.89 & 249.84 & -51.88 & 183.80 & 8.51 & $\mathrm{~s}$ \\
9 & 138.56 & 297.00 & 81.22 & 271.69 & 4.47 & $\mathrm{p}$ \\
10 & 138.68 & 140.71 & -34.27 & 114.23 & 3.60 & $\mathrm{p}$ \\
\hline \hline
\end{tabular}




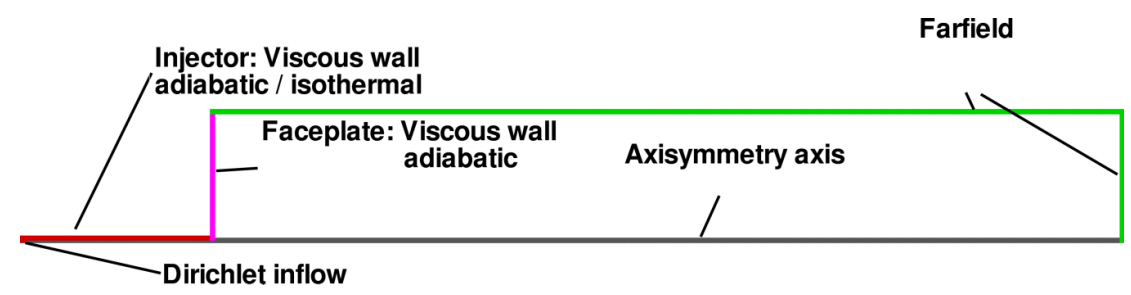

FIG. 13. Numerical domain and boundary conditions.

jets which exhibit no constant density core. Such a case can be simply calculated with CFD. The exact thermal conditions of the experiments are unknown. While an extensive numerical parameter study might identify thermal boundary conditions which match experimental findings, this does not necessarily mean that these thermal conditions were actually present in the experiment. Thus, reproduction of the experiment is not sought here. Instead, an exemplary computation is carried out. The goal is to determine whether non-dense-core injection undergoing a thermal disintegration can be reproduced when injector heat flux is accounted for. The literature of CFD simulations has been discussed. Reviewing the discussed literature with the new insight at hand shows that all authors assume adiabatic injectors.

\section{B. Numerical setup}

Computations are carried out using the DLR TAU Code. ${ }^{43,44}$ TAU is a hybrid grid, finite volume, compressible CFD code which solves the integral form of the Euler and Navier-Stokes equations. The real gas model is presented in Banuti and Hannemann. ${ }^{45,46,48}$ Real gas properties are calculated in a preprocessing step and stored in lookup-tables in order to improve numerical efficiency. Here, Younglove's Modified Benedict Webb Rubin (MBWR) equation of state ${ }^{31}$ has been employed to determine nitrogen real gas properties. A Spalart-Allmaras ${ }^{47}$ turbulence model is used.

The computational domain is sketched in Fig. 13. It encompasses the $2.2 \mathrm{~mm}$ diameter, $90 \mathrm{~mm}$ long injector attached to a chamber, as used in the experiments. As the experiment is axisymmetric, the computational domain is $2 \mathrm{D}$. In order to provide a defined steady state where the cryogenic nitrogen enters room temperature surroundings, the numerical setup is chosen to differ from the experimental setup: instead of computing a closed chamber that slowly floods with cryogenic nitrogen, an open surrounding is modeled. The faceplate remains a viscous wall. The outer wall boundary condition is replaced with a farfield boundary condition. The distance from the injector $(27 \mathrm{D}$ in radial direction, $181 \mathrm{D}$ in axial direction) is well beyond the $6 \mathrm{D}$ radial and $40 \mathrm{D}$ axial distance specified by Zong et al. ${ }^{38}$ as being sufficiently far away to minimize boundary influence on the free jet. The structured grid is comprised of 220000 cells to ensure sufficient resolution. Injector inflow properties are imposed as block profile Dirichlet boundary conditions at the beginning of the injector. Injection condition is a temperature of $128 \mathrm{~K}$, a density of $514 \mathrm{~kg} / \mathrm{m}^{3}$, and a pressure of $5.98 \mathrm{MPa}$.

Two computations are carried out: the first using the conventional adiabatic injector and the second with an isothermal injector at ambient temperature.

\section{Results}

Figure 14 shows the resulting density and temperature distributions in the near injector region for an adiabatic injector. A dense potential core is clearly visible, interaction of the jet with its surroundings begins in the shear layer. As expected, slight variations in temperature around the pseudo-boiling point lead to significant variations in density.

Allowing for heat transfer inside the injector changes the injection pattern substantially. Figure 15 compares density distributions of the adiabatic and the isothermal injector. In the latter, a thermal boundary layer builds up prior to injection. The density of the jet is diminished, starting from the walls and progressing towards the center. The adiabatic dense core reaches to $X / D \approx 7$, while it ends some five $D$ before entering the chamber in the heated case. 


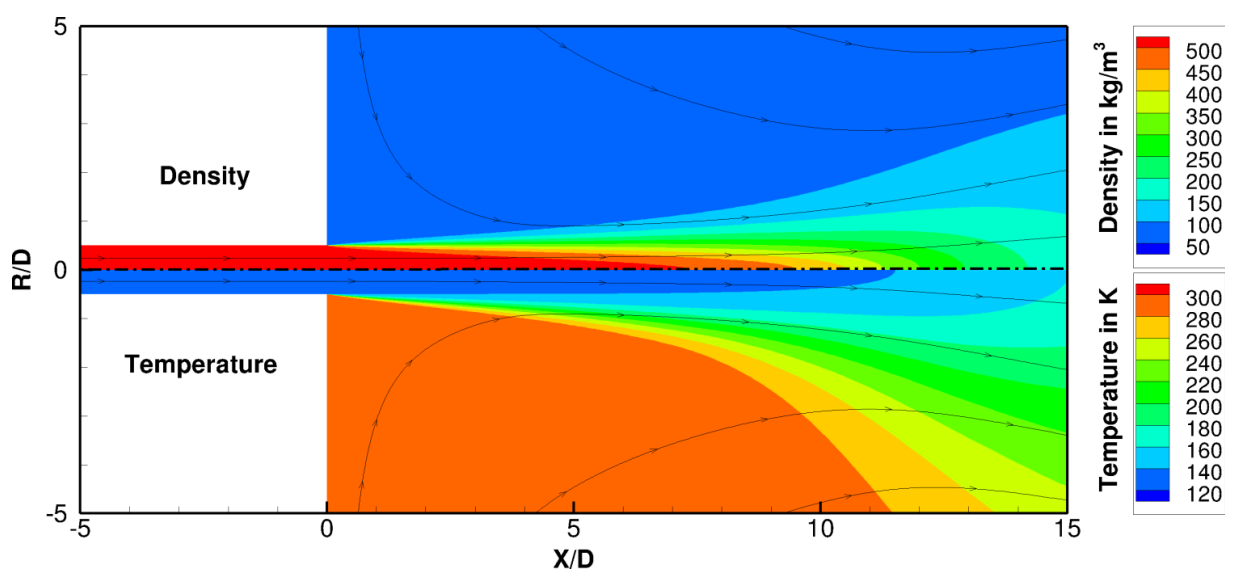

FIG. 14. Density (top) and temperature (bottom) distributions for adiabatic injector.

Figure 16(a) shows axial density profiles of both computations, starting inside the injector at $X / D=-5$. While the adiabatic computation shows the classical dense core, the isothermal injector shows a sloped density profile starting inside the injector.

Radial density distributions for two axial positions are shown in Fig. 16(b), right at the injector exit $X / D=0$, and downstream at $X / D=1.2$. In the adiabatic case, the jet essentially enters the chamber with a temperature and density top hat profile; a slight variation in density can be attributed to viscous dissipation in the boundary layer. Downstream, the shear layer has smoothed the distribution between $0.35<R / D<0.65$. The density profile of the isothermal injector instead is fully thermally developed, the temperature rise reaches all the way to the center. At $X / D=0$, the density has dropped from the initial value of $514.0 \mathrm{~kg} / \mathrm{m}^{3}$ to $480 \mathrm{~kg} / \mathrm{m}^{3}$. With the profile fully thermally developed at $X / D=0$, the shear layer does not immediately affect the flow inside the jet. It does, however, smooth the interface to the surroundings similarly to what is seen in the adiabatic case.

\section{INTERPRETATION OF DISINTEGRATION MODES}

At this point, joint experimental, numerical, and theoretical evidence suggests the existence and relevance of a thermal disintegration mechanism. This deserves a more thorough discussion.

Figure 17 presents the topology of thermal disintegration, based on the variation of fluid properties across pseudo-boiling as shown in Fig. 6. Figure 17(a) indicates how the stream changes its thermodynamic properties upon injection through an injector with wall heat transfer. Initially in a

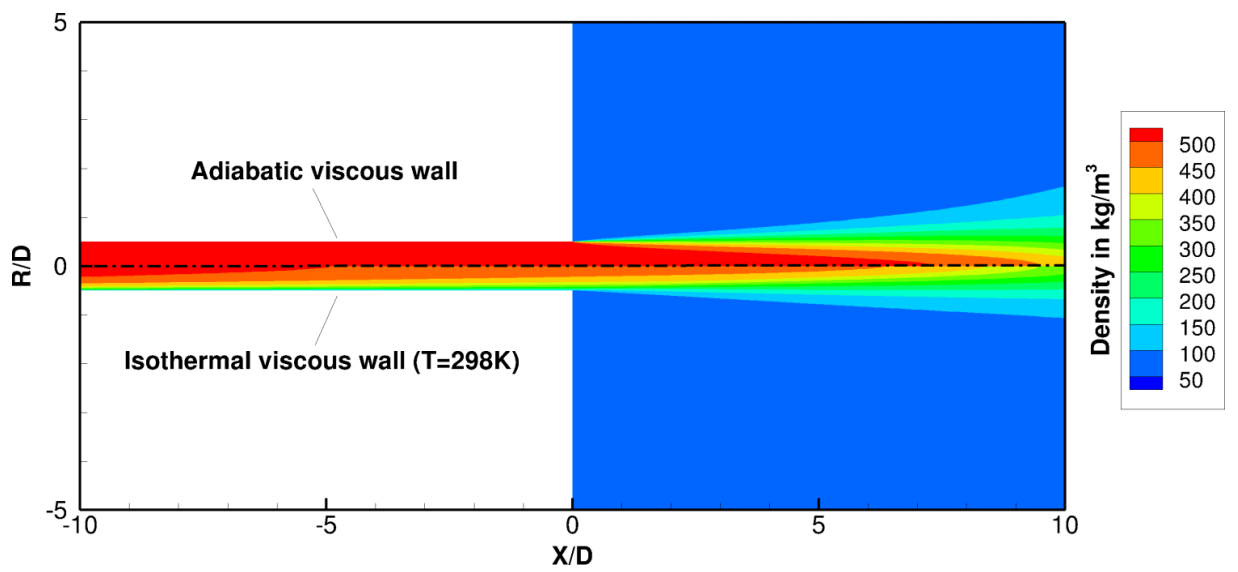

FIG. 15. Comparison of density distributions for adiabatic (top) and isothermal (bottom) injector wall. 


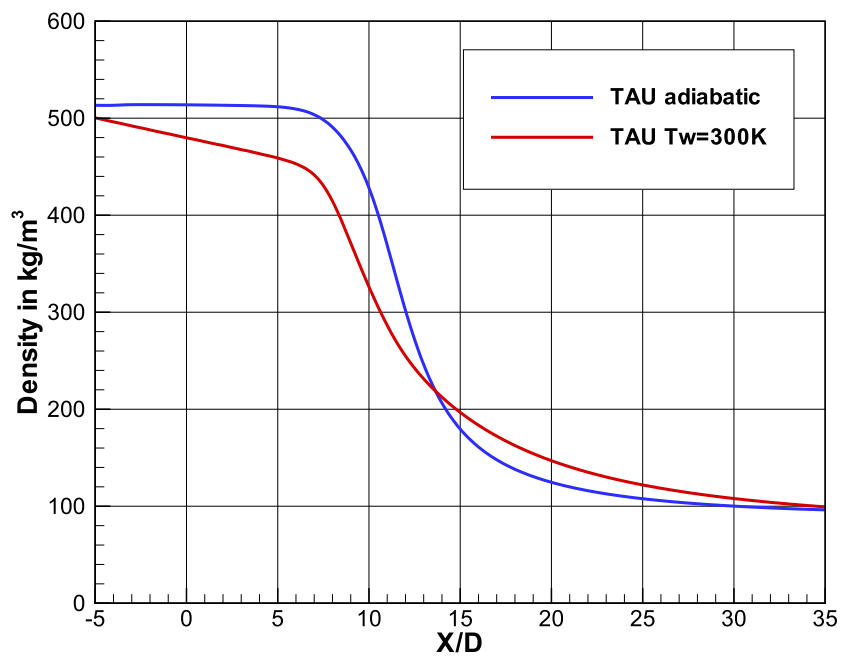

(a)

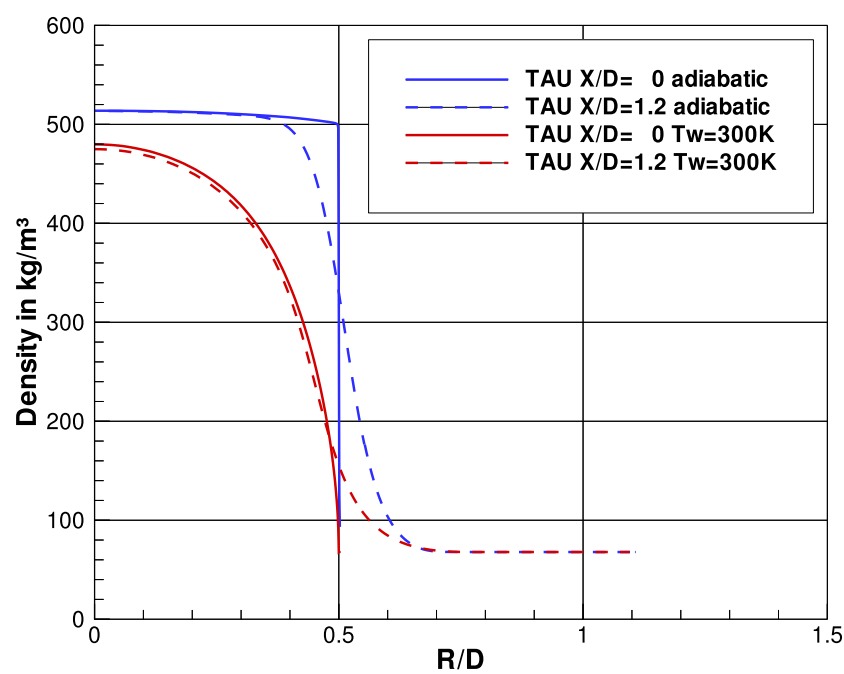

(b)

FIG. 16. Comparison of density profiles for adiabatic and isothermal injector walls. (a) Axial density profiles. (b) Radial density profiles.

liquid-like state, heating leads to the development of a thermal boundary layer which propagates towards the centerline. Density gradients are maximal where the fluid reaches the pseudo-boiling temperature $T_{\mathrm{pb}}$ but remain large in a transitional layer of a certain temperature range. After sufficient heating, thermal expansion is reduced to a degree comparable to an ideal gas, where the temperature sensitivity is substantially reduced. Finally, mixing with warm ambient gas leads to a further rapid reduction of the density at the centerline when it has been reached by the expanding shear layer.

Here, "potential core" no longer signifies an unperturbed region of constant properties. Instead, it merely denotes the region which remains unaffected by the chamber conditions.

Accordingly, different "core lengths" may be defined in relation to the injection plane: the length of the liquid like core $L_{\mathrm{L}}$, the length of the dense core reaching to the maximal density gradient $L_{\mathrm{D}}$, the end of the transitional region $L_{\mathrm{T}}$, and the classical potential core length $L_{\mathrm{C}}$ at which the shear layer has expanded to the centerline. 


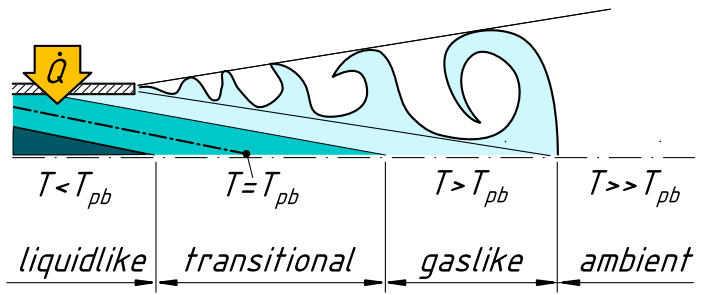

(a)

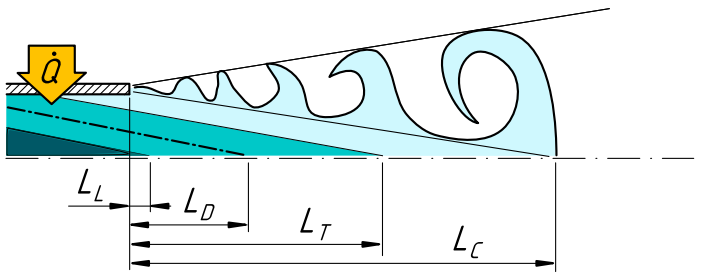

(b)

FIG. 17. Structure of thermal disintegration in supercritical injection. (a) Different thermodynamic zones in thermal disintegration. (b) Definition of disintegration lengths for supercritical injection.

Necessarily, $L_{\mathrm{L}}<L_{\mathrm{D}}<L_{\mathrm{T}}$ when heat is added, as the fluid successively undergoes the thermodynamic state change illustrated in Fig. 8. Actual values will vary dependent on the fluid state and the character of the heat transfer. The observed disintegration modes may be explained in terms of this variation.

Figure 18(a) illustrates the dense core, as obtained experimentally in cases 3 and 7, being consistent with classical mechanical break-up theory and obtained in simulations with adiabatic injector walls. This pattern can be expected when heat transfer in the injector is not sufficient to affect the liquid-like core, i.e., $L_{\mathrm{L}}=L_{\mathrm{C}}$. A constant property pattern may also emerge when heat
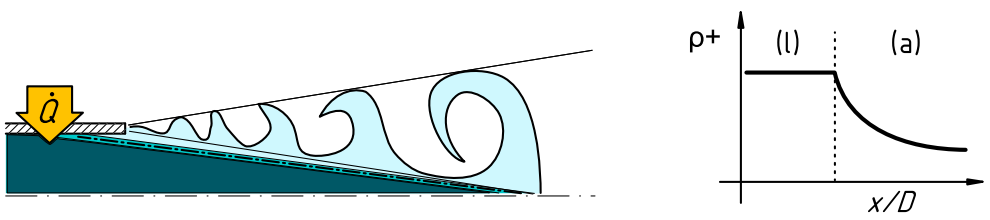

(a)
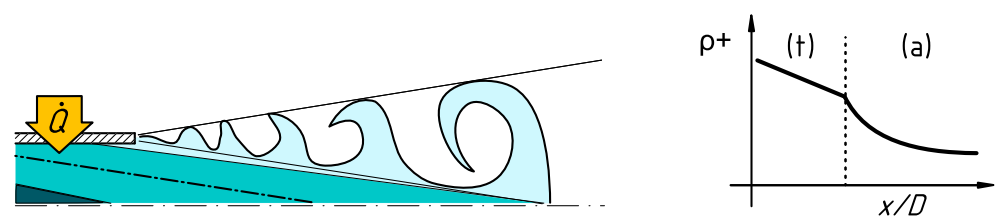

(b)
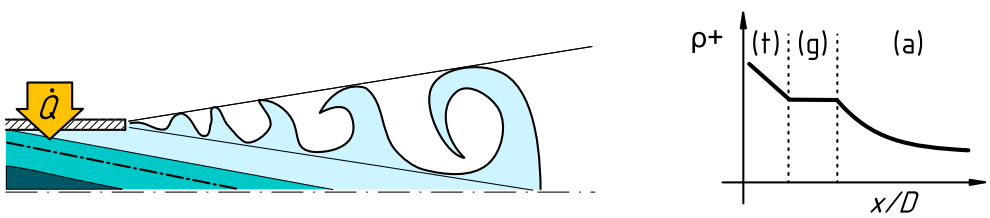

(c)

FIG. 18. Structure of observed thermal disintegration modes. Regions with reference to Fig. 17(a): (1)iquidlike, (t)ransitional, (g)aslike, (a)mbient. (a) Dense core. (b) Slope. (c) Plateau. 


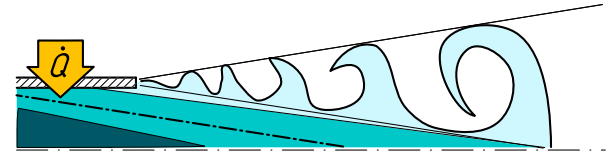

(a)

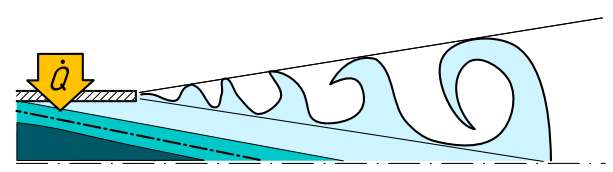

(b)
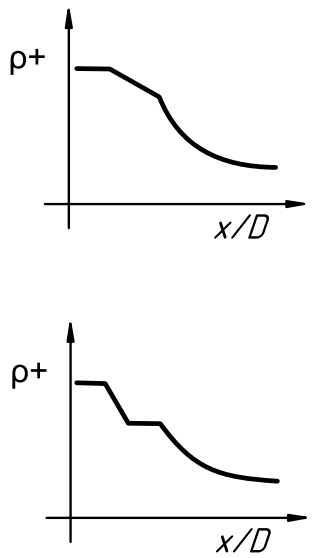

FIG. 19. Structure of additional thermal disintegration modes. (a) Core slope. (b) Core plateau.

transfer is so large that all of the fluid has reached a homogeneous thermally insensitive gas-like state prior to injection, $L_{\mathrm{T}}<0$.

Figure 18(b) shows the sloped core, as found in cases 4 and 8 . The pattern has been obtained numerically when finite heat transfer is accounted for by imposing isothermal injector walls. No liquid core is visible, $L_{\mathrm{L}}<0$. The transition to a gas-like state is not complete as the shear layer enforces ambient mixing: A state change not complete at $L_{\mathrm{C}}$ is found to be dominated by entrainment of the surrounding fluid, $L_{\mathrm{T}} \leq L_{\mathrm{C}}$.

The plateau type core Fig. 18(c) is observed in cases 5, 6, 9, and 10. It has not been calculated in the proof-of-concept CFD study discussed in this paper. However, applying the structural disintegration model introduced in Fig. 17, a plausible explanation may be given: Again, no liquid core is visible, $L_{\mathrm{L}}<0$. The density in the transitional region drops upon entering the chamber. The transition to a less thermally sensitive gas-like state is complete before the shear layers meet at the centerline, $L_{\mathrm{T}}<L_{\mathrm{C}}$, allowing an intermediate potential region to be formed. The plateau type pattern is only observed at the low injection velocity cases with corresponding low mass flow. This means that the required power to reach pseudo-boiling for the whole stream is reduced while the residence time in the injector is increased. Both characteristics enhance heating of the stream.

Following the rationale, two more cases can be hypothesized which have not yet been observed. They represent variations of the slope and the plateau type with a liquid core protruding into the chamber, $L_{\mathrm{L}}>0$. These modes are visualized in Fig. 19.

\section{SUMMARY/CONCLUSION}

In this paper we point out a systematic discrepancy between experiment and theory of supercritical injection: We identify three distinct break-up modes in data from the literature, two of which show an instant decline of density right upon entering the chamber. This behavior cannot be explained in the framework of the current, i.e., mechanical, state of theory. Furthermore, a literature review reveals that so far no CFD computation was successful in predicting subsided dense cores.

We remedy this dilemma by introducing the concept of a "thermal disintegration" mechanism. It is unique to supercritical fluids due to their sensitivity of density on moderate heat addition across the "pseudo-boiling-line," a supercritical continuation of the vapor-pressure curve. During pseudo-boiling, the fluid undergoes a thermodynamic state change from a supercritical liquid-like to a gas-like state-resembling subcritical vaporization.

In the experiments, the energy required to heat the injected fluid to the pseudo-boiling state correlates with the existence of a dense core; the jet is more likely to undergo thermal disintegration when it is close to pseudo-boiling during injection. 
Thus, heat transfer in the injector serves as a substitute for mechanical interaction in that it may shorten the dense core. This heat flux may act in the injector, prior to injection.

By capturing the subsided core in CFD, we could demonstrate that this phenomenon can be predicted by standard real gas solvers. It is however important that the thermal boundary condition be chosen appropriately: allowing for heat transfer in the injector replaced the dense potential core with a subsided density core.

${ }^{1}$ C. D. Boer, G. Bonar, S. Sasaki, and S. Shetty, in SAE World Congress E Exhibition, SAE 2013-01-0257 (SAE, Detroit, USA, 2013).

${ }^{2}$ M. Habiballah, M. Orain, F. Grisch, L. Vingert, and P. Gicquel, "Experimental studies of high-pressure cryogenic flames on the Mascotte facility," Combust. Sci. Technol. 178, 101 (2006).

${ }^{3}$ S. Candel, M. Juniper, G. Singla, P. Scouflaire, and C. Rolon, "Structure and dynamics of cryogenic flames at supercritical pressure," Combust. Sci. Technol. 178, 161 (2006).

${ }^{4}$ B. Chehroudi, D. Talley, and E. Coy, AIAA Paper No. 99-0206, 1999.

${ }^{5}$ M. Oschwald, J. Smith, R. Branam, J. Hussong, A. Schik, B. Chehroudi, and D. Talley, "Injection of fluids into supercritical environments," Combust. Sci. Technol. 178, 49 (2006).

${ }^{6}$ B. Chehroudi, "Physical hypothesis for the combustion instability in cryogenic liquid rocket engines," J. Propul. Power 26, 1153 (2010).

${ }^{7}$ H. Rehab, E. Villermaux, and E. Hopfinger, "Flow regimes of large-velocity-ratio coaxial jets," J. Fluid Mech. 345, 357 (1997).

${ }^{8}$ G. Abramovich, The Theory of Turbulent Jets (MIT Press, Cambridge, 1963).

${ }^{9}$ E. Villermaux, "Mixing and spray formation in coaxial jets," J. Propul. Power 14, 807 (1998).

${ }^{10} \mathrm{~J}$. Lasheras and E. Hopfinger, "Liquid jet instability and atomization in a coaxial gas stream," Annu. Rev. Fluid Mech. 32 275 (2000).

${ }^{11}$ B. Chehroudi, D. Talley, and E. Coy, "Visual characteristics and initial growth rates of round cryogenic jets at subcritical and supercritical pressures," Phys. Fluids 14, 850 (2002).

12 M. Oschwald and A. Schik, "Supercritical nitrogen free jet investigated by spontaneous Raman scattering," Exp. Fluids 27, 497 (1999).

${ }^{13}$ M. Oschwald and M. Micci, "Spreading angle and centerline variation of density of supercritical nitrogen jets," Atomization Sprays 11, 91 (2002).

${ }^{14}$ R. Branam and W. Mayer, "Length scales in cryogenic injection at supercritical pressure," Exp. Fluids 33, 422 (2002).

${ }^{15}$ T. Kim, Y. Kim, and S.-K. Kim, "Numerical study of cryogenic liquid nitrogen jets at supercritical pressures," J. Supercrit. Fluids 56, 152 (2011).

${ }^{16}$ H. Müller, C. Niedermeier, M. Jarczyk, M. Pfitzner, S. Hickel, and N. Adams, in Proceedings of the EUCASS Conference (EUCASS, Munich, Germany, 2013).

${ }^{17}$ C. Niedermeier, M. Jarczyk, S. Hickel, N. Adams, and M. Pfitzner, AIAA Paper 2013-2950, 2013.

${ }^{18}$ T. Schmitt, L. Selle, B. Cuenot, and T. Poinsot, "Large-eddy simulation of transcritical flows," C. R. Mec. 337, 528 (2009).

19 T. Schmitt, L. Selle, A. Ruiz, and B. Cuenot, "Large-eddy simulation of supercritical-pressure round jets," AIAA J. 48, 2133 (2010).

20 J.-P. Hickey, P. Ma, M. Ihme, and S. Thakur, AIAA Paper 2013-4071, 2013.

${ }^{21}$ L. Cutrone, P. de Palma, G. Pascazio, and M. Napolitano, AIAA Paper 2008-4567, 2008.

${ }^{22}$ L. Cutrone, P. de Palma, G. Pascazio, and M. Napolitano, "A RANS flamelet-progress-variable method for computing reacting flows of real-gas mixtures," Comput. Fluids 39, 485 (2010).

${ }^{23}$ G. Cheng and R. Farmer, AIAA Paper 2002-785, 2002.

${ }^{24}$ M.-M. Jarczyk and M. Pfitzner, AIAA Paper 2012-1270, 2012.

${ }^{25} \mathrm{H}$. Terashima and M. Koshi, "Unique characteristics of cryogenic nitrogen jets under supercritical pressures," J. Propul. Power 29, 1328 (2013).

${ }^{26}$ E. Antunes, A. Silva, and J. Barata, AIAA Paper 2015-0469, 2014.

${ }^{27}$ W. Mayer, J. Telaar, R. Branam, G. Schneider, and J. Hussong, "Raman measurements of cryogenic injection at supercritical pressure," Heat Mass Transfer 39, 709 (2003).

${ }^{28}$ R. Branam and W. Mayer, "Characterization of cryogenic injection at supercritical pressure," J. Propul. Power 19, 342 (2003).

${ }^{29}$ W. Mayer, B. Ivancic, A. Schik, and U. Hornung, "Propellant atomization and ignition phenomena in liquid oxygen/gaseous hydrogen rocket combustors," J. Propul. Power 17, 794 (2001).

${ }^{30}$ W. Mayer, J. Telaar, R. Branam, G. Schneider, and J. Hussong, AIAA Paper 2001-3275, 2001

${ }^{31}$ B. A. Younglove, "Thermophysical properties of fluids. I. Argon, Ethylene, Parahydrogen, Nitrogen, Nitrogen Trifluoride, and Oxygen,” J. Phys. Chem. Ref. Data 11 (Suppl. 1), 1-353 (1982).

32 D. Davis and B. Chehroudi, AIAA Paper 2004-1330, 2004.

${ }^{33}$ D. Davis and B. Chehroudi, "Measurements in an acoustically driven coaxial jet under sub-, near-, and supercritical conditions," J. Propul. Power 23, 364 (2007).

${ }^{34}$ NIST Chemistry Webbook, edited by P. Linstrom and W. Mallard, NIST Standard Reference Database Number 69 (National Institute of Standards and Technology, Gaithersburg, MD 20899, 2013), http://webbook.nist.gov.

${ }^{35}$ J. Newman and T. Brzustowski, "Behavior of a liquid jet near the thermodynamic critical region," AIAA J. 9, 1595 (1971)

${ }^{36}$ B. Chehroudi, "Recent experimental efforts on high-pressure supercritical injection for liquid rockets and their implications," Int. J. Aerosp. Eng. 2012, 121802. 
${ }^{37}$ R. Span, E. Lemmon, R. Jacobsen, W. Wagner, and A. Yokozeki, in NIST Chemistry WebBook, edited by P. Linstrom and W. Mallard, NIST Standard Reference Database Number 69 (National Institute of Standards and Technology, Gaithersburg, MD 20899, 2010), http://webbook.nist.gov (retrieved 12 November 2010).

${ }^{38}$ N. Zong, H. Meng, and V. Yang, "A numerical study of cryogenic fluid injection and mixing under supercritical conditions," Phys. Fluids 16, 4248 (2004).

${ }^{39}$ N. Kafengauz and M. Federov, "Pseudoboiling and heat transfer in a turbulent flow," Inzh.-Fiz. Zh. 14, 923 (1968).

40 D. Banuti, "Crossing the Widom-line-Supercritical pseudo-boiling," J. Supercrit. Fluids 98, 12 (2015).

${ }^{41}$ D. Banuti, "Thermodynamic analysis and numerical modeling of supercritical injection," $\mathrm{Ph} . \mathrm{D}$. thesis, University of Stuttgart, 2015

42 D. Banuti and K. Hannemann, AIAA Paper 2011-5620, 2011.

${ }^{43}$ S. Karl, "Numerical investigation of a generic scramjet configuration,” Ph.D. thesis, University of Dresden, 2011 http:// nbn-resolving.de/urn:nbn:de:bsz:14-qucosa-68695.

${ }^{44}$ T. Gerhold, Notes on Numerical Fluid Mechanics and Multidisciplinary Design (NNFM) (Springer, 2005), Vol. 89, pp. 81-92.

45 D. Banuti and K. Hannemann, AIAA Paper 2013-4068, 2013.

${ }^{46}$ D. Banuti and K. Hannemann, AIAA Paper 2014-3791, 2014

${ }^{47}$ P. Spalart and S. Allmaras, AIAA Paper No. AIAA-92-0439, 1992.

${ }^{48}$ D. Banuti, V. Hannemann, K. Hannemann, and B. Weigand, "An efficient multi-fluid-mixing model for real gas reacting flows in liquid propellant rocket engines," Combust. Flame (in press). 